\title{
Experimental identification of the radiation resistance matrix
}

Joseph Milton, ${ }^{1}$ Jordan Cheer, ${ }^{1,}$ a) and Steve Daley ${ }^{1}$

Institute of Sound and Vibration Research, University of Southampton, Southampton, SO17 1BJ

(Dated: 16 April 2019) 
The radiation resistance matrix allows for the calculation of structurally radiated sound power using a series of measured structural responses. Currently, estimating the radiation resistance matrix requires precise modelling of the structure which, for practical structures, can lead to estimation errors. This paper presents two methods for identifying the radiation resistance matrix for a structure using measurable structural and acoustic responses and the solution of an inverse problem. Although well suited to practical, complex structures, to allow the accuracy of the proposed methods of identifying the radiation resistance matrix to be reliably validated, they are compared with the theoretical radiation resistance matrix for a flat plate in an infinite baffle. It is shown through a simulation-based study that the accuracy of the proposed identification methods depends on the number of structural and acoustic sensors and structural forces used in the identification process. The proposed identification methods are then implemented experimentally to identify the radiation resistance matrix for a flat plate. The results demonstrate that an accurate estimate of the sound power can be obtained using the experimentally identified radiation resistance matrix using the two proposed methods, and the limits on the two methods are discussed.

a)J.Cheer@soton.ac.uk 


\section{INTRODUCTION}

Active Structural Acoustic Control (ASAC) has been proposed as an effective, lightweight solution to structure-borne sound radiation problems. Generally speaking, ASAC uses an array of structural control actuators to minimise the acoustic pressure measured at an array of error microphones, located in the radiated sound field. The application of such a system can be limited, however, owing to the fact that it is not always practical to position error microphones in the radiated sound field. In previous work, to overcome this problem, a number of strategies have been developed for use in both ASAC and Active Noise Control (ANC), that allow the sound field to be estimated from remotely located error sensors ${ }^{1-5}$.

Controlling the pressure radiated from a structure at discrete points may be sufficient in certain applications, however, many ASAC systems have focused on controlling the radiated sound power, which when minimised ensures a global reduction. Measuring the radiated sound power directly is not straightforward, however, there have been a number of publications that propose different approaches to controlling the radiated sound power from a structure using only structural measurements ${ }^{6-8}$. One such approach, that has been proposed relatively recently, uses the Weighted Sum of Spatial Gradients (WSSG) to attempt to control the structural radiation ${ }^{9,10}$. The WSSG can be measured using a closely spaced array of four accelerometers and it has been shown that the WSSG provides a uniform measure across the surface of a plate and, therefore, is insensitive to the location of the WSSG sensor and does not require advance knowledge of the structure ${ }^{9}$. The WSSG control method has been shown through both simulations and experiments to provide effective control of the 
sound power radiated from a flat plate $^{9}$. More recently, the method has been extended to a cylindrical structure ${ }^{10}$, where it has been shown to provide close to optimal sound power control in numerical simulation, but has some limitations in an experimental implementation due to discrepancies in the structural properties and assumed boundary conditions ${ }^{10}$. Nevertheless, the WSSG method does potentially provide an ASAC method that is convenient to implement in practice requiring only a small number of error sensors, even if multiple WSSG sensors are required to improve the practical robustness.

Alternative approaches to implementing ASAC using only structural sensors include the use of the radiation resistance matrix ${ }^{7,11}$, which completely describes the radiation from a structure in terms of its radiation $\operatorname{modes}^{6}$. The radiation resistance matrix has previously been calculated using a variety of methods, including analytical or numerical modelling using an elemental lumped parameter approximation, for example ${ }^{11-13}$. These modelling-based methods, however, often rely on specific assumptions about the radiating structure. For example, that the sound radiation can be approximated by the radiation from an array of point monopole sources, which may lead to inaccuracies when considering more complex practical structures. In order to overcome these difficulties, various methods of identifying the radiation resistance matrix experimentally have previously been proposed ${ }^{14,15}$. Koopmann and Fahnline for example, developed a method to measure the elements of the radiation resistance matrix using a bespoke measurement probe, named the resistance probe ${ }^{14}$. This method relies on the probe generating a known volume velocity from a loudspeaker positioned on the surface of the structure. To achieve accuracy at higher frequencies the probe must be sufficiently small so that it represents a small area of the radiating structure, how- 
ever, this conflicts with the requirement to be able to generate sufficiently high volume velocity at low frequencies. These conflicting requirements mean that the method has been shown to have a low frequency limit of around $200 \mathrm{~Hz}$ and, therefore, may have limited applicability in situations where active control would be appropriate. More recently, Berkhoff et al. presented a method whereby the radiation resistance matrix is calculated by solving an inverse problem, which uses the responses measured between a number of structural forces and distributions of both structural velocity and acoustic pressure measurements ${ }^{15}$. This method has been shown to be effective experimentally, however, as the acoustic pressures are used to calculate the radiated sound power, in order to accurately identify the radiation resistance matrix, the radiating structure must be located in a free-field acoustic environment and the pressure measurements must be taken in the far-field. This may be infeasible for many practical structures, which cannot be relocated to a free-field acoustic environment. Therefore, in this paper a method to identify the radiation resistance matrix is proposed that does not require a free-field acoustic environment or far-field pressure measurements. The proposed method can thus be used, for example, when the structure is in situ.

Two formulations of the proposed method are presented which both rely on the solution of an inverse problem. In the first formulation, the proposed method uses the responses measured between a distribution of structural forces and an array of structural velocity, and near-field acoustic pressure and particle velocity measurements to identify the radiation resistance matrix. In the second formulation, it is assumed that the particle velocity immediately in front of the surface of the structure is equal to the velocity of the structure itself 
and, therefore, only structural velocity and near-field acoustic pressure measurements are used to identify the radiation resistance matrix.

The structure of this paper is as a follows: Section II introduces the theoretical background for the radiation resistance matrix and, using the amplitudes of an array of elemental radiators, the radiation resistance matrix is calculated for a flat plate in an infinite baffle for reference. Section III then presents the formulation of the first radiation resistance matrix identification method. The accuracy of the proposed method is then assessed via comparison to the theoretical radiation resistance matrix formulated in Section II, for a simulated flat plate in an infinite baffle. A simulation based investigation into how the accuracy of the proposed radiation resistance matrix is affected by the number of forces and sensors used in the identification process is then carried out and this is followed by an experimental validation of the method. In Section IV, the second identification method is formulated and its accuracy is assessed through both simulations and an experimental validation. Finally, Section V draws conclusions based on the presented results.

\section{FORMULATION OF THE RADIATION RESISTANCE MATRIX USING EL- EMENTAL RADIATORS}

In this section, a well established, theoretical radiation resistance matrix formulation is presented. Initially, the theory of the formulation is detailed, followed by an example of the radiation resistance matrix calculated for a flat plate in an infinite baffle. This established structure will be used in the following sections to validate the proposed identification methods. 


\section{A. Theory}

A lumped parameter model can be used to model the acoustic radiation from a vibrating structure as equivalent to that of a simple point source, at low frequencies, where the acoustic wavelength is much larger than the characteristic dimension of the vibrating structure. In this form, the acoustic field depends only on the volume velocity of the source ${ }^{14}$. This approach can be extended to model larger structures and/or higher frequencies by subdividing the surface of the radiating structure into a number of elements ${ }^{12,14}$.

In this formulation, it is assumed that the sound radiated by a structure is due to a number of elementary radiators ${ }^{6,11}$. The acoustic pressure immediately in front of each radiating element can be related to the complex velocity of each element at a single frequency according to the impedance relationship

$$
\mathbf{p}_{s}=\mathbf{Z v}
$$

where $\mathbf{v}$ is the vector of complex elemental structural velocities, $\mathbf{p}_{s}$ is the vector of corresponding acoustic pressures on the surface of the structure and $\mathbf{Z}$ is the matrix of specific acoustic impedances, which incorporates point and transfer impedance terms over the grid of elements. Assuming at this point that the acoustic radiation from each element can be approximated as a monopole source radiating into half space, defined by the infinite baffle ${ }^{6,11}$, the impedance between the $i^{\text {th }}$ and $j^{\text {th }}$ elements of the matrix $\mathbf{Z}$ can be defined as

$$
Z_{i j}(\omega)=\frac{\mathrm{j} \omega \rho_{0} A_{e}}{2 \pi R_{i j}} e^{-\mathrm{j} k R_{i j}}
$$

where $\omega$ is angular frequency, $\rho_{0}$ is the density of air, $A_{e}$ is the area of each element, $k$ is the wavenumber and $R_{i j}$ is the distance between the centres of the $i^{t h}$ and $j^{\text {th }}$ elements. 
The sound power radiated by the elemental array described above can be defined in terms of the vector of structural velocities and acoustic pressures as

$$
W=\left(\frac{A_{e}}{2}\right) \mathbb{R e}\left[\mathbf{p}_{s}^{H} \mathbf{v}\right]
$$

By substituting Eq. 1 into Eq. 3, for the vector of pressures, the radiated sound power can be written as $^{11}$

$$
W=\left(\frac{A_{e}}{2}\right) \mathbb{R e}\left[\mathbf{v}^{H} \mathbf{Z} \mathbf{v}\right]=\left(\frac{A_{e}}{4}\right) \mathbf{v}^{H}\left[\mathbf{Z}+\mathbf{Z}^{H}\right] \mathbf{v}=\mathbf{v}^{H} \mathbf{R} \mathbf{v}
$$

where, in the final expression, the impedance matrix, $\mathbf{Z}$, is assumed to be symmetric due to acoustic reciprocity and the real, symmetric, positive definite radiation resistance matrix is defined as $\mathbf{R}=\left(A_{e} / 2\right) \mathbb{R e}[\mathbf{Z}]$. It can be seen from the final expression of Eq. 4, that the radiation resistance matrix can be used to calculate the radiated sound power using only structural velocities and, therefore, can be used in an ASAC system that only requires structural error sensors rather than acoustic sensors ${ }^{11}$.

\section{B. Baffled plate}

In the following, the radiation resistance matrix is calculated according to Section II, for a flat, rectangular plate in an infinite baffle. For elemental models, a common rule of thumb is to use six elements per wavelength ${ }^{6}$. In the following, a $0.414 \mathrm{~m}$ by $0.314 \mathrm{~m}$ plate is divided into ten elements along the $x$-axis and eight elements along the $y$-axis, as shown in Fig. 1 . When the upper frequency of interest is $1 \mathrm{kHz}$, this provides at least eight elements per the shortest radiated acoustic wavelength and will thus provide an accurate model of the plate radiation. 


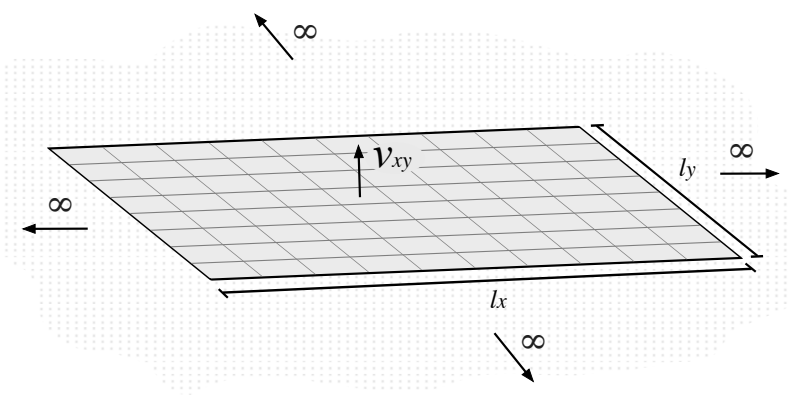

FIG. 1. Geometry of the $0.414 \mathrm{~m} \times 0.314 \mathrm{~m}$ plate subdivided into a $10 \times 8$ grid of elements in an infinite baffle.

As noted above, the radiation resistance matrix completely describes the acoustic radiation from a structure. It is possible to gain insight into the physics behind this sound radiation via an eigen-decomposition of the radiation resistance matrix which can be expressed $\mathrm{as}^{6}$,

$$
\mathbf{R}=\mathbf{Q}^{T} \Lambda \mathbf{Q}
$$

where $\mathbf{Q}$ is the matrix of eigenvectors, in which each row contains the amplitudes of each radiation mode, and $\boldsymbol{\Lambda}$ is the diagonal matrix of eigenvalues, which are proportional to the radiation efficiencies of the radiation modes. The radiation efficiencies of the first six radiation modes of the baffled plate shown in Fig. 1 have been plotted in Fig. 2 using the eigenvalues, $\lambda_{r}$, of the radiation resistance matrix. From this plot it can be seen that the magnitude of the radiation efficiencies vary slowly with frequency, increasing in magnitude as the frequency increases. It can also be seen that at low frequencies the radiation response will be dominated by the first radiation mode, whilst with increasing frequency the contribution of the higher order modes will become increasingly important. Through the decomposition of the radiation resistance matrix, surface plots of the velocity distribution of the six lowest 
order radiation modes of the plate, when excited at $39 \mathrm{~Hz}$, have been plotted in Fig. 3. From these plots it can be seen that the first and most efficiently radiating mode is the piston mode; this is followed by two rocking modes, a torsional mode and two saddle modes. Visualising the radiation modes in this way could help to tailor the positioning of control actuators and sensors when designing an ASAC system, or even lead to the design of radiation based modal actuators as $\mathrm{in}^{16,17}$. It is important to note that although the radiation modes are frequency dependant, within the range considered $(0 \mathrm{~Hz}$ to $1 \mathrm{kHz})$ there is only a slight variation in the mode shapes of the dominant, lower order modes ${ }^{6}$.

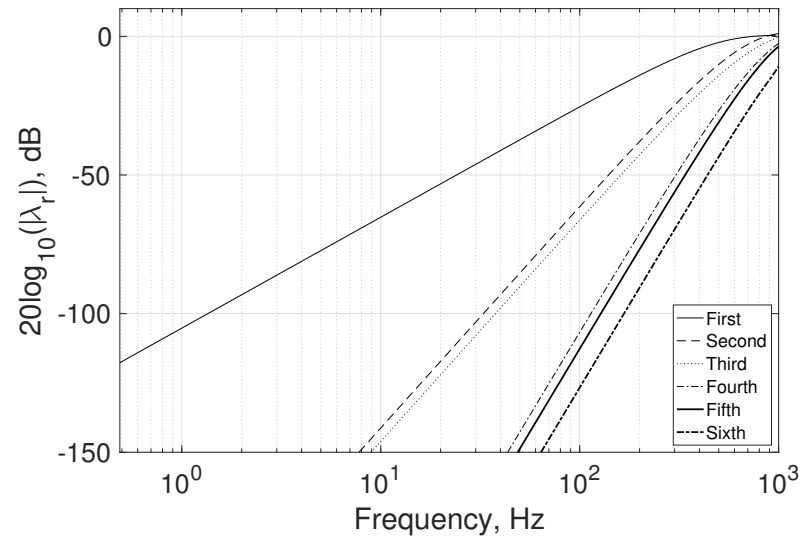

FIG. 2. Eigenvalues of the six lowest order modes of the radiation resistance matrix.

\section{INVERSE ESTIMATION OF THE RADIATION RESISTANCE MATRIX US- ING P-U MEASUREMENTS}

In the section, the first of the proposed methods of estimating the radiation resistance matrix via the solution of an inverse problem is formulated and investigated via both simulations and experiments. This method uses a series of near-field particle velocity and acoustic 


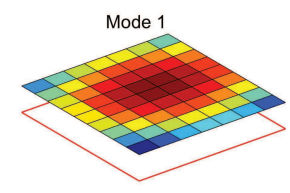

(a)

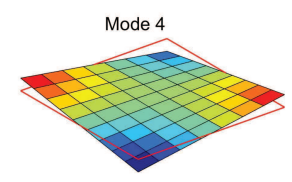

(d)

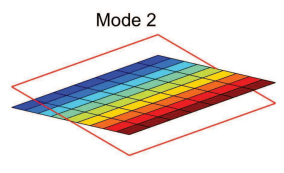

(b)

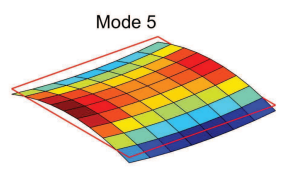

(e)

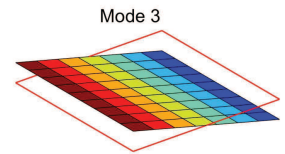

(c)

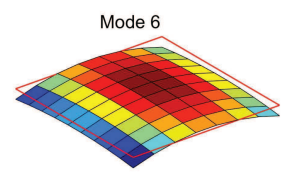

(f)

FIG. 3. (color online) Six lowest order radiation modes of the plate when excited at $39 \mathrm{~Hz}$.

\section{A. Formulation}

The sound power radiated from a structure can be expressed in terms of the vectors of particle velocities, $\mathbf{u}$, and acoustic pressures, $\mathbf{p}$, measured on a virtual surface enclosing the structure as

$$
W=\left(\frac{A_{e}}{2}\right) \mathbb{R e}\left\{\mathbf{p}^{H} \mathbf{u}\right\}
$$

where $A_{e}$ is the area over which the particle velocities and acoustic pressures are measured, divided by the number of measurement positions ${ }^{18}$. It is possible to express the acoustic pressures and particle velocities in terms of the structural responses as

$$
\mathbf{p}=\widetilde{\mathbf{H}}_{p} \mathbf{v} \quad \text { and } \quad \mathbf{u}=\widetilde{\mathbf{H}}_{u} \mathbf{v}
$$


where $\mathbf{v}$ is a vector of structural velocities measured on the surface of the structure and $\widetilde{\mathbf{H}}_{p}$ and $\widetilde{\mathbf{H}}_{u}$ are the transfer response matrices between the measured structural velocities and the acoustic pressures and particle velocities respectively. It should be noted that the structural response could alternatively be expressed in terms of accelerations or displacements without any implications on the proposed method, which may have practical benefits. Substituting Eqs. 7 into Eq. 6 for the vectors of acoustic pressures and particle velocities, the radiated sound power may be written in terms of the structural responses as

$$
W=\left(\frac{A_{e}}{2}\right) \mathbb{R e}\left\{\mathbf{v}^{H} \widetilde{\mathbf{H}}_{p}^{H} \widetilde{\mathbf{H}}_{u} \mathbf{v}\right\} .
$$

Expanding this in terms of its real and imaginary parts allows a simplification that gives

$$
W=\left(\frac{A_{e}}{4}\right) \mathbf{v}^{H}\left[\Xi^{H}+\Xi\right] \mathbf{v}=\mathbf{v}^{H} \widehat{\mathbf{R}} \mathbf{v}
$$

where $\Xi=\widetilde{\mathbf{H}}_{p}^{H} \widetilde{\mathbf{H}}_{u}$ and the radiation resistance matrix is defined as $\widehat{\mathbf{R}}=\left(A_{e} / 2\right) \mathbb{R e}\left[\widetilde{\mathbf{H}}_{p}^{H} \widetilde{\mathbf{H}}_{u}\right]$. It is not possible to measure the matrices $\widetilde{\mathbf{H}}_{p}$ and $\widetilde{\mathbf{H}}_{u}$ directly, as each of the structural responses cannot be independently driven and are only controllable via a fully coupled transfer response matrix ${ }^{19}$. It is possible, however, to estimate these matrices via the solution of an inverse problem ${ }^{19,20}$.

The vector of structural responses, $\mathbf{v}$, can be expressed in terms of a distribution of forces, f, and a structural transfer response matrix as

$$
\mathbf{v}=\mathbf{H}_{s} \mathbf{f}
$$

where $\mathbf{H}_{s}$ is the matrix of transfer responses between the distribution of forces and the measured structural responses, which can be measured directly. The relationships between 
the transfer responses, $\widetilde{\mathbf{H}}_{p}, \widetilde{\mathbf{H}}_{u}$ and $\mathbf{H}_{s}$, and the vectors of forces, $\mathbf{f}$, structural velocities, $\mathbf{v}$, acoustic pressures, $\mathbf{p}$, and acoustic particle velocities, $\mathbf{u}$, are summarised by the block diagram shown in Fig. 4. From this block diagram, or by substituting Eq. 10 into Eqs. 7, the vectors of acoustic pressures and particle velocities can be expressed as

$$
\mathbf{p}=\widetilde{\mathbf{H}}_{p} \mathbf{H}_{s} \mathbf{f}=\mathbf{H}_{p} \mathbf{f} \quad \text { and } \quad \mathbf{u}=\widetilde{\mathbf{H}}_{u} \mathbf{H}_{s} \mathbf{f}=\mathbf{H}_{u} \mathbf{f} .
$$

The transfer matrices $\widetilde{\mathbf{H}}_{p}$ and $\widetilde{\mathbf{H}}_{u}$ can then be obtained using the directly measurable transfer response matrices, via the solution of two corresponding inverse problems given as

$$
\widetilde{\mathbf{H}}_{p}=\mathbf{H}_{p} \mathbf{H}_{s}^{\dagger} \quad \text { and } \quad \widetilde{\mathbf{H}}_{u}=\mathbf{H}_{u} \mathbf{H}_{s}^{\dagger},
$$

where the superscript $\dagger$ denotes the pseudo-inverse operator. When the number of structural measurements is equal to the number of forces, so that $\mathbf{H}_{s}$ is a square matrix, Eqs. 12 can be solved using the direct matrix inversion $\mathbf{H}_{s}^{-1}$. However, if the structural response matrix is not square, then the solution to the inverse problem must be calculated via the pseudoinverse. The particular solution to the pseudo-inverse is dependant on the dimensions of the structural response matrix and, therefore, the relative numbers of forces and structural sensors used to measure it. Section III B 1 presents an investigation into how changing the number of forces and sensors affects the accuracy of the radiation resistance matrix. The specific solution to the pseudo-inverse will also be discussed in more detail.

\section{B. Simulation based investigation}

In this section, the radiation resistance matrix is estimated, via the proposed inverse method, for a simulated flat, rectangular plate of dimensions, $l_{x}=0.414$ and $l_{y}=0.314$, as 


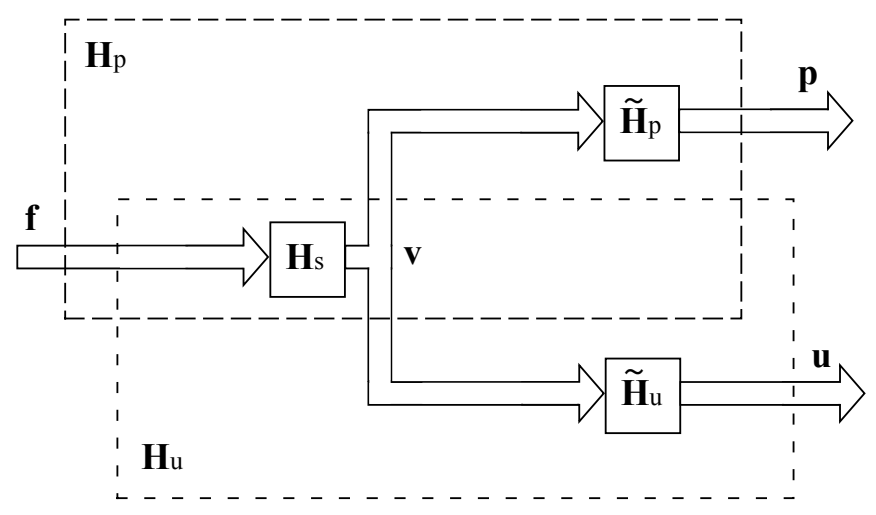

FIG. 4. Block diagram showing the relationships between the vectors of forces, $\mathbf{f}$, structural velocities, $\mathbf{v}$, acoustic pressures, $\mathbf{p}$ and acoustic particle velocities, $\mathbf{u}$, in terms of the directly measurable transfer response matrices $\mathbf{H}_{p}, \mathbf{H}_{u}$ and $\mathbf{H}_{s}$ and the transfer response matrices $\widetilde{\mathbf{H}}_{p}$ and $\widetilde{\mathbf{H}}_{p}$ that can be identified via Eq. 12 .

described in Section II B. A diagram of the simulated plate setup used to obtain the transfer responses used in the formulation is shown in Fig. 5, and this includes an example of the distribution of structural forces and sensors and the acoustic pressure and particle velocity sensors used in the identification procedure.

In order to assess the performance of the proposed identification method, the accuracy of the sound power estimated using the radiation resistance matrix is compared to the directly evaluated sound power. The modelled plate is excited by an acoustic plane wave incident at $45^{\circ}$ in the horizontal plane and $45^{\circ}$ in the vertical plate, which approximates a diffuse field excitation ${ }^{6}$. The sound power radiated by the plate is then calculated using both the structural responses of the plate with the estimated radiation resistance matrix and directly from the pressures and particle velocities according to Eq. 6 . 


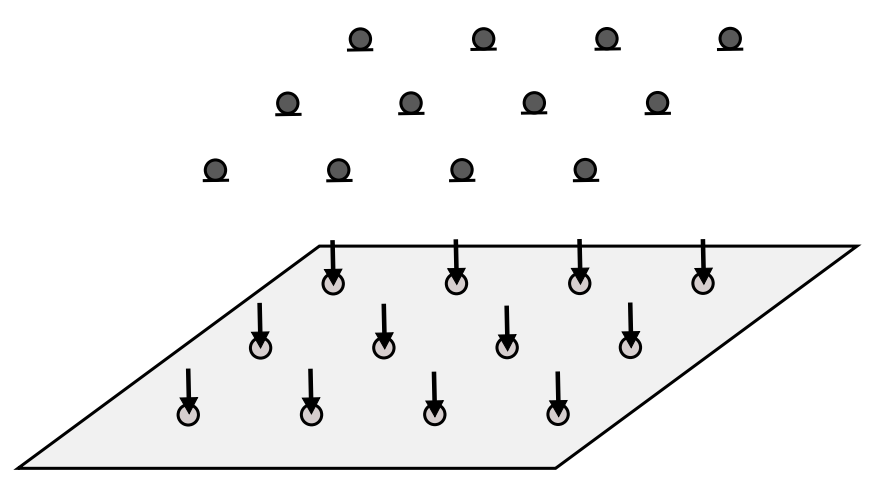

FIG. 5. Diagram of the plate with a distribution of independent forces (arrows), collocated with an array of structural velocity sensors (light circles), beneath an array of near-field acoustic sensors (dark circles).

The estimated radiation resistance matrix, $\widehat{\mathbf{R}}$, is initially calculated using the modelled transfer responses between a uniform distribution of $10 \times 8$ forces to an equal number of structural velocities and near-field acoustic pressures and particle velocities. This means that the matrix of structural responses, $\mathbf{H}_{s}$, is square and the direct matrix inversions can be used in Eqs. 12 during the estimation procedure. It is worth reiterating that this forcesensor arrangement gives a minimum of eight forces and sensors per the radiated acoustic wavelength at $1 \mathrm{kHz}$, but it is also worth noting that this provides around 2.4 forces and sensors per structural bending wavelength at the upper frequency of interest.

Fig. 6 shows the radiated sound power when the plate is excited by an incident acoustic plane wave, as defined above. The sound power calculated using the estimated radiation resistance matrix, $\widehat{\mathbf{R}}$, is plotted along with the directly evaluated sound power, which is calculated according to Eq. 6, using the uniform array of $10 \times 8$ particle velocities and acoustic pressures, evaluated $10 \mathrm{~mm}$ above the surface of the plate. These results show that 
the sound power estimate is consistent with the directly evaluated sound power when the same number of both forces and sensors are used to estimate the radiation resistance matrix as acoustic sensors are used to evaluate the sound power.

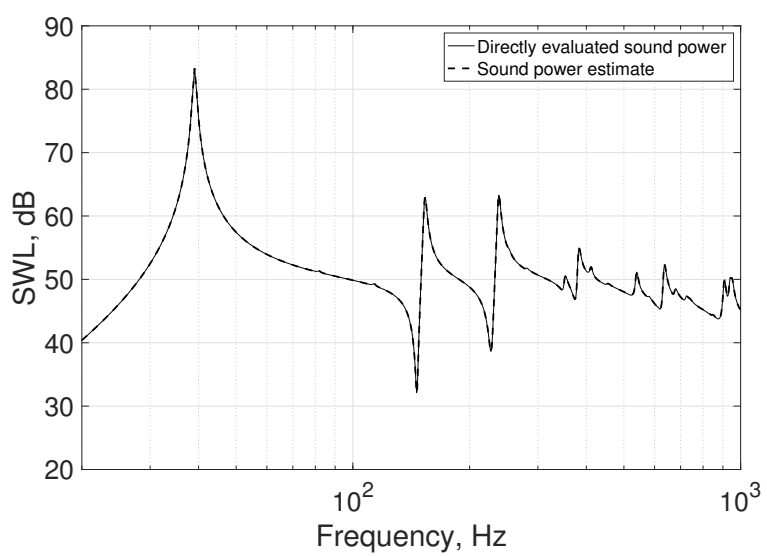

FIG. 6. Sound power radiated by the plate when excited by an incident acoustic plane wave. The solid line shows the directly evaluated sound power and the dashed black line shows the sound power calculated using the estimated radiation resistance matrix, $\widehat{\mathbf{R}}$.

\section{The effect of the number of forces and sensors used in the estimation of the radiation resistance matrix}

It has been demonstrated above that the proposed method of estimating the radiation resistance matrix is able to accurately estimate the radiated sound power when large and equal numbers of both forces and sensors are used in the identification procedure. However, in practice it may not always be possible to use such large and square arrays and, therefore, this Section will present a simulation based investigation into the accuracy of the proposed radiation resistance matrix identification procedures when the numbers of sensors and/or 
forces are both modified and made unequal. To assess the accuracy of the identification method as the number of forces and/or sensors is altered, the sound power calculated using the proposed radiation resistance matrix is compared to the directly evaluated sound power.

In the first instance, the number of structural sensors used to estimate the radiation resistance matrix has been decreased whilst the number of acoustic sensors and structural forces remains fixed at eight per the shortest acoustic wavelength, which is approximately $0.34 \mathrm{~m}$ at $1 \mathrm{kHz}$. This means that the matrix describing the structural transfer responses, $\mathbf{H}_{s}$, is not square, and therefore, the estimation procedure cannot utilise the direct matrix inversions. Instead the inverse problems defined by Eqs. 12, must be solved via the pseudoinverse. The form of the pseudo-inverse required is dependant on whether the number of forces, $L_{f}$, is greater or less than the number of structural sensors, $L_{s}$. In the following, the number of forces will always be greater than the number of structural sensors and, therefore, the matrix $\mathbf{H}_{s}$, which has dimensions $\left(L_{s} \times L_{f}\right)$ will be full row rank. This means that the solution to the inverse problem is obtained using the right-sided pseudo-inverse, which is given as

$$
\mathbf{H}_{s}^{\dagger}=\mathbf{H}_{s}^{H}\left(\mathbf{H}_{s} \mathbf{H}_{s}^{H}\right)^{-1} .
$$

Fig. 7 shows the sound power estimated using the radiation resistance matrix as the number of structural sensors was reduced, plotted along with the directly evaluated sound power. It can be seen from Fig. 7, that reducing the number of structural sensors used to calculate the radiation resistance matrix to seven per the shortest acoustic wavelength, which corresponds to two per the shortest structural bending wavelength, has negligible effect on the estimated sound power over the presented frequency range. Reducing the number of 
structural sensors to five per the shortest acoustic wavelength, which corresponds to around 1.2 per the shortest structural bending wavelength, begins to introduce some small errors at the upper end of the presented frequency range. As the number of structural sensors reduces further, to approximately three per the shortest acoustic wavelength, it can be seen from the results that errors begin to appear at higher frequencies. The results in this case are still accurate up to around $400 \mathrm{~Hz}$, because the structural sensor arrangement provides around 8 sensors per the acoustic wavelength or approximately 2 per the structural bending wavelength at this frequency. Reducing the number of sensors further, to approximately one per the shortest acoustic wavelength, leads to the errors becoming large and occurring at lower frequencies.

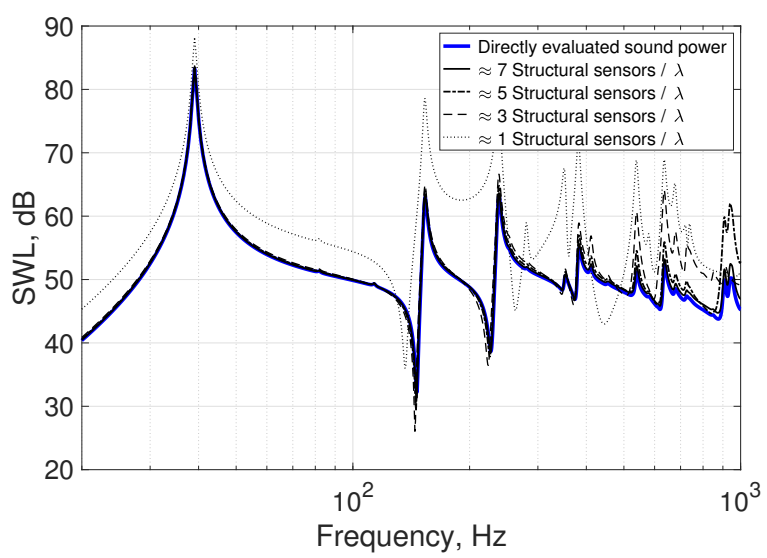

FIG. 7. (color online) Sound power estimated using the radiation resistance matrix as the number of structural sensors used in the identification is decreased. The blue solid line shows the directly evaluated sound power, the solid, dot-dashed, dashed and dotted black lines show the sound power estimate when seven, five, three and one structural sensors per the shortest acoustic wavelength were used in the identification of the radiation resistance matrix, respectively. 
In the following, the number of forces used to identify the radiation resistance matrix is reduced and the number of structural and acoustic sensors used remains fixed at eight per the shortest acoustic wavelength. Again, as matrix the $\mathbf{H}_{s}$ is not square, the inverse problems must be solved via the pseudo-inverse as defined by Eqs. 12. In this case, the number of forces will always be less than the number of structural sensors and, therefore, the matrix $\mathbf{H}_{s}$ will be full column rank. This means that the solution to the inverse problem should be obtained using the left sided pseudo-inverse, which is given as

$$
\mathbf{H}_{s}^{\dagger}=\left(\mathbf{H}_{s}^{H} \mathbf{H}_{s}\right)^{-1} \mathbf{H}_{s}^{H} .
$$

Fig. 8 shows the radiated sound power calculated using the estimated radiation resistance matrix as the number of forces used in the estimation is reduced, plotted along with the directly evaluated sound power. From Fig. 8 it can clearly be seen that as the number of forces used to identify the radiation resistance matrix is reduced, the accuracy of the sound power estimate decreases. In particular, it can be seen that with seven forces per the shortest acoustic wavelength, the sound power estimate is accurate over the full frequency range presented. When the number of forces is reduced to five per the shortest acoustic wavelength a similar level of accuracy is obtained, but with some small errors at higher frequencies. However, when reducing the number of forces used in the identification to three per the shortest acoustic wavelength, which corresponds to less than one per the shortest structural bending wavelength, the accuracy of the sound power estimate is limited at higher frequencies. When the number of forces per the shortest acoustic wavelength is reduced to approximately one, more errors are introduced across the entire frequency range, as expected. 


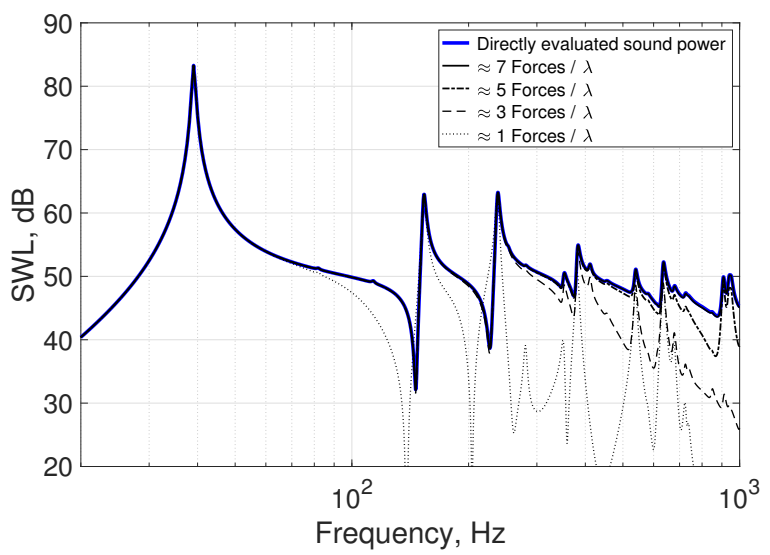

FIG. 8. (color online) Sound power estimated using the radiation resistance matrix as the number of forces used in the identification is decreased. The blue solid line shows the directly evaluated sound power, the solid, dot-dashed, dashed and dotted black lines show the sound power estimate when seven, five, three and one forces per the shortest acoustic wavelength were used in the identification of the radiation resistance matrix, respectively.

Finally, the effect of reducing the number of acoustic sensors has been investigated. In the following simulation results, the number of structural sensors and forces was fixed at eight per the shortest acoustic wavelength, whilst the number of uniformly distributed particle velocity and acoustic pressure measurements was decreased. In this case, the matrix $\mathbf{H}_{s}$ is square and, therefore, the direct inverse solution can be used. Fig. 9 shows the sound power calculated using the estimated radiation resistance matrix as the number of acoustic measurements is reduced. From these results it can be seen that the accuracy of the sound power estimate is largely unaffected as the number of acoustic measurements is decreased. That said, some errors are introduced into the sound power estimate when there are only 1.5 sensors per acoustic wavelength. This observation is consistent with the Nyquist sampling 
theorem and it is evident that an accurate estimation is provided when there are at least 2 acoustic sensors per the shortest acoustic wavelength. This is in contrast to the results presented in Figs. 7 and 8, because the speed of sound in the structure is much higher than in air and the associated wavelengths are thus much shorter. This means that a larger number of forces and structural sensors is required in relation to the acoustic wavelength.

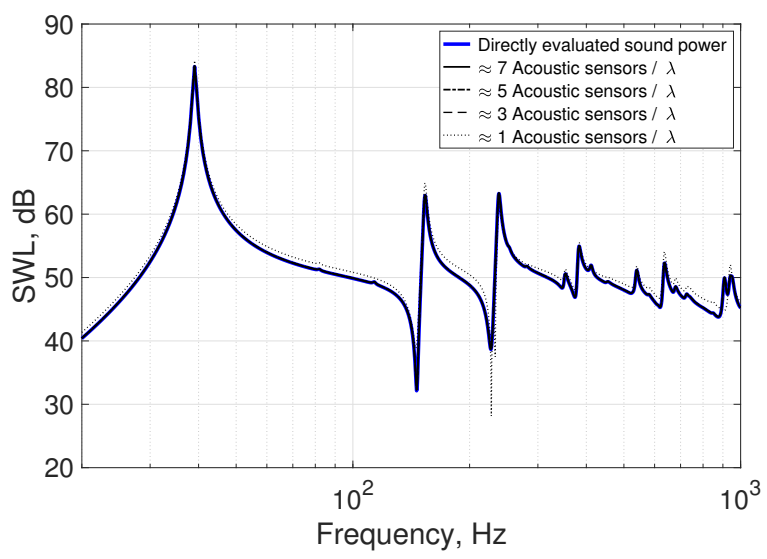

FIG. 9. (color online) Sound power estimated using the radiation resistance matrix as the number of acoustic measurements used in the identification is decreased. The blue solid line shows the directly evaluated sound power, the solid, dot-dashed, dashed and dotted black lines show the sound power estimate when seven, five, three and one acoustic measurements per the shortest acoustic wavelength were used in the identification of the radiation resistance matrix, respectively.

Following the simulation investigation carried out above, it is clear that a reduction in the number of forces and structural sensors results in a rapid reduction in the accuracy of the sound power estimate. The number of acoustic sensors also effects the accuracy, but, due to the longer wavelength in air compared to that in the structure, the effects are smaller. In Section III C, the proposed radiation resistance matrix identification method is implemented 
in practice to obtain a sound power estimate for an acoustically excited aluminium plate. In order to carry out this experiment, an appropriate number of structural and acoustic sensors and forces should be chosen. It is obvious that for the most accurate sound power estimate, a very large number of sensors and forces should be used, however, this would increase the cost of conducting the procedure. On the other hand, using less sensors and forces would reduce the cost of the procedure but at a detriment to the accuracy of the sound power estimation. Therefore, to strike a balance between an overly populated system, in which the number of actuators, sensors and cabling required would make the system extremely costly, and the accuracy of the sound power estimate, three forces and three structural and acoustic sensors per the shortest acoustic wavelength has been chosen. According to the results presented in Figs. 7,8 and 9, this is expected to provide an accurate estimation up to around $400 \mathrm{~Hz}$, but it is important to initially assess within the simulation environment the impact of reducing both forces, and structural and acoustic sensors simultaneously. The proposed configuration corresponds to 12 forces and 12 structural and acoustic sensors uniformly distributed over the plate and the accuracy of the sound power estimate achieved when using this arrangement to identify the radiation resistance matrix for the simulated plate is shown in Fig. 10. These results show that the estimate identifies the majority of the radiating modes of the plate across the given frequency range, however, above approximately $400 \mathrm{~Hz}$ errors appear in the level of the sound power estimate. This is as expected from the results presented in Figs. 7,8 and 9 and provides two clear frequency ranges where the estimation is expected to be accurate $(f<400 \mathrm{~Hz})$ and suffer from errors $(f>400 \mathrm{~Hz})$. 


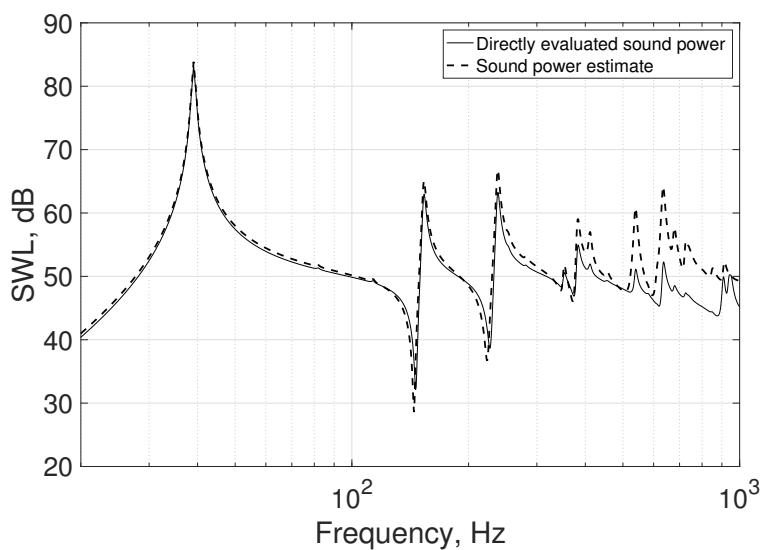

FIG. 10. Sound power radiated by the simulated plate when acoustically excited with broadband noise. The solid line shows the measured sound power and the dashed line shows the sound power calculated using the proposed radiation resistance matrix $\widehat{\mathbf{R}}$ with 3 forces, and 3 structural and acoustic sensors per the shortest acoustic wavelength.

\section{Experimental Validation}

Based on the simulation study carried out above, an experimental rig has been built to validate the proposed methods of estimating the radiation resistance matrix. In the experimental setup, which is shown in Fig. 11, the forces have been provided by 12 lightweight (29.6 g) inertial actuators, the structural sensors have been provided by 12 accelerometers, approximately collocated with each actuator, and the acoustic pressure and particle velocity have been measured using a Microflown $\mathrm{p}-\mathrm{u}$ probe. To measure the transfer responses required for the estimation of the radiation resistance matrix, each of the inertial actuators was driven independently with broadband noise to excite the plate and the structural response was measured by the array of accelerometers, and the acoustic pressure and particle 
velocity responses were measured above each accelerometer position, approximately $10 \mathrm{~mm}$ from the surface of the plate.

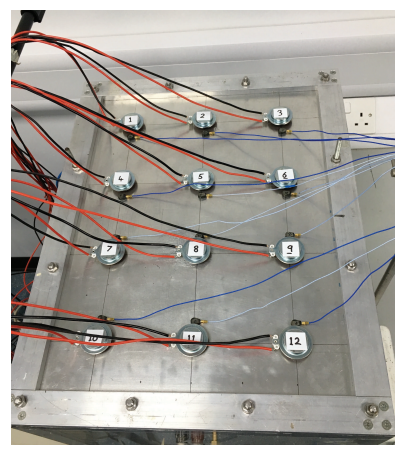

(a)

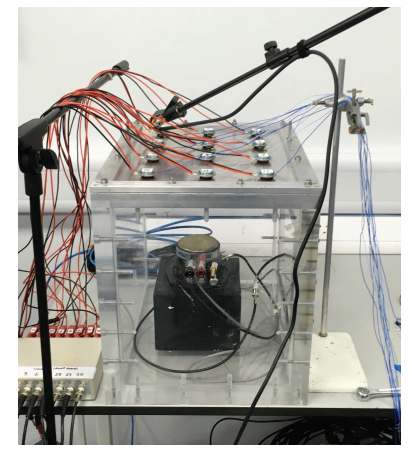

(b)

FIG. 11. (color online) Photograph of the experimental setup used to measure the radiation resistance matrix for the plate. The 12 actuators and approximately collocated accelerometers can be seen on the plate and the p-u probe can be seen in $11(\mathrm{~b})$

A loudspeaker enclosed in the sealed cavity below the plate, shown in Fig. 11(b), was used to excite the plate acoustically. The radiated sound power was then calculated directly using the measured pressure and particle velocities as in Eq. 6, and using the radiation resistance matrix estimated using the proposed method, formulated in Section III A.

Fig. 12 shows the sound power estimate plotted along with the directly evaluated sound power. It should be noted that for this plate the breathing mode is at approximately $100 \mathrm{~Hz}$ and the peak at around $50 \mathrm{~Hz}$ is due to the resonance of the actuators. From these results it can be seen that, at frequencies below around $400 \mathrm{~Hz}$, the identified radiation resistance matrix is able to estimate the radiated sound power relatively accurately. The four lowest radiating modes, which are within this bandwidth, are identified in both frequency and amplitude. At $400 \mathrm{~Hz}$ and above, the accuracy of the estimation reduces, as expected 
from the simulation results presented in Fig. 10. Initially, the resonance frequencies are identified, but the sound power level estimation is inaccurate. Then, at higher frequencies, the resonance frequencies themselves become poorly estimated.

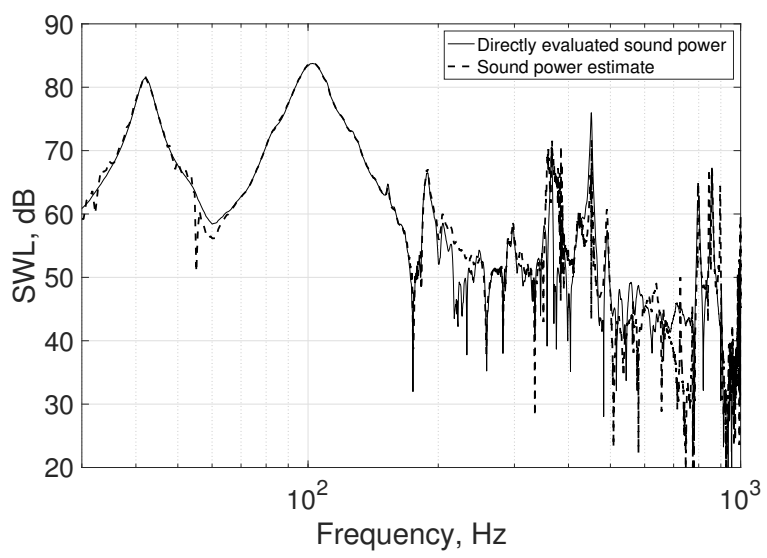

FIG. 12. Sound power radiated by the plate when acoustically excited with broadband noise. The solid line shows the measured sound power and the dashed line shows the sound power calculated using the estimated radiation resistance matrix $\widehat{\mathbf{R}}$.

\section{INVERSE ESTIMATION OF THE RADIATION RESISTANCE MATRIX US- ING PRESSURE AND STRUCTURAL VELOCITY MEASUREMENTS}

In practical applications, it may be beneficial to avoid the requirement to measure the acoustic particle velocity, which requires a costly measurement probe. This can be achieved by assuming that the velocity of the vibrating structure is equal to the acoustic particle velocity immediately in front of it. Based on this assumption, in this section, an alternative approach to identifying the radiation resistance matrix using only near-field acoustic pressure and structural velocity measurements is presented. Following the formulation of the 
proposed estimation method, a simulation based investigation and experimental validation are carried out.

\section{A. Formulation}

As suggested above, by making the approximation that the structural velocity, $\mathbf{v}$, is equal to the particle velocity, $\mathbf{u}$, immediately in front of it, the matrix of responses between structural velocities and acoustic particle velocities, $\widetilde{\mathbf{H}}_{u}$, becomes equal to an identity matrix. In this case, following the derivation presented in Section III A, the radiated sound power can be approximated as

$$
\begin{aligned}
W & \approx\left(\frac{A_{e}}{2}\right) \operatorname{Re}\left[\mathbf{v}^{H} \widetilde{\mathbf{H}}_{p}^{H} \mathbf{v}\right] \\
& \approx\left(\frac{A_{e}}{4}\right) \mathbf{v}^{H}\left[\widetilde{\mathbf{H}}_{p}^{H}+\widetilde{\mathbf{H}}_{p}\right] \mathbf{v}=\mathbf{v}^{H} \widehat{\mathbf{R}}_{p} \mathbf{v},
\end{aligned}
$$

where the radiation resistance matrix in this case is $\widehat{\mathbf{R}}_{p}=\left(A_{e} / 2\right) \mathbb{R e}\left[\widetilde{\mathbf{H}}_{p}\right]$. As detailed in Section III A, the transfer response matrix, $\widetilde{\mathbf{H}}_{p}$, cannot be directly measured and must be estimated through the solution of an inverse problem, which is given by the first part of Eq. 12.

It should be noted that the approximation in Eq. 15 relies on the assumption that the structural velocities are equivalent to the particle velocities measured at the same points as the acoustic pressures. Thus, if it were possible to measure the pressures on the surface of the structure, as assumed in the theoretical formulation in Section II, then this assumption would be perfectly accurate. However, in practice, the pressures will be measured at a finite 
distance from the surface of the structure and this assumption will only be approximate; this will be demonstrated in the following section.

\section{B. Simulation based investigation}

Using the simulation environment described in Section IIIB, the performance of the radiation resistance matrix identified using the method outlined in Section IV A has been validated. For conciseness, this investigation is only presented using a single arrangement of forces and sensors, because the same trends are observed for this method as were shown in Section III B. Therefore, in this case, the $10 \times 8$ arrays of forces, structural velocities and acoustic pressures were used to estimate the radiation resistance matrix.

Fig. 13 shows the sound power radiated by the plate, along with the sound power estimated using the radiation resistance matrix, $\widehat{\mathbf{R}}_{p}$, when the array of pressure measurements are evaluated at various distances from the plate. When the pressures are evaluated at $0.01 \mathrm{~m}$ from the surface of the plate, it can be seen that the estimated radiation resistance matrix, $\widehat{\mathbf{R}}_{p}$, provides an accurate measure of the radiated sound power. In this case, therefore, the approximation that the structural velocity is equal to the acoustic particle velocity is reliable over the full frequency range presented. However, it can also be seen from the results presented in Fig. 13 that as the array of pressure sensors is moved away from the surface of the plate, the accuracy of the sound power estimate reduces. This indicates that the approximation becomes increasingly less accurate as the pressure measurements are moved away from the radiating surface and the upper frequency at which the approximation is reliable can be related to the acoustic wavelength. 


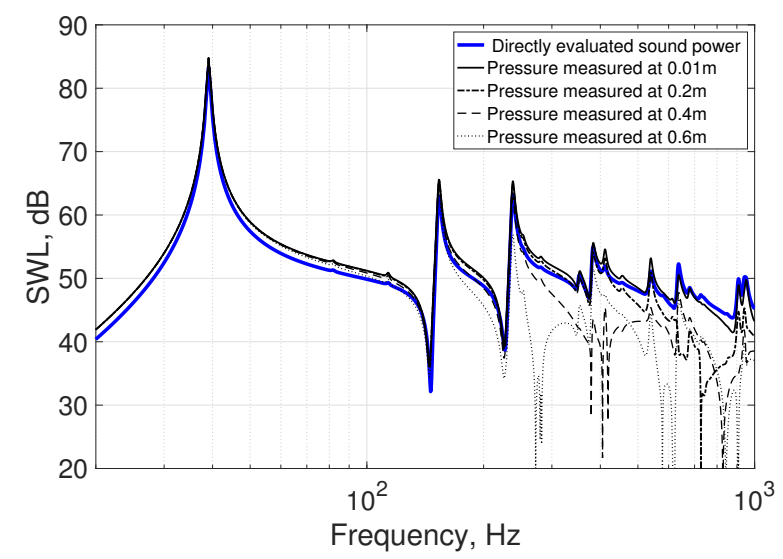

FIG. 13. (color online) Sound power radiated by the plate when excited by an incident acoustic plane wave. The blue solid line shows the directly evaluated sound power, the solid, dot-dashed, dashed and dotted black lines show the sound power estimated using the estimated radiation resistance matrix, $\widehat{\mathbf{R}}_{p}$, when the pressures are measured at $0.01,0.2,0.4$ and $0.6 \mathrm{~m}$ respectively.

\section{Experimental validation}

In this section, the sound power radiated from the acoustically excited plate shown in Fig. 11 has been estimated using the radiation resistance matrix, $\widehat{\mathbf{R}}_{p}$, which is identified as formulated in Section IV A. This validation has been carried out using the same $4 \times 3$ experimental setup shown in Fig. 11, with the pressures measured at a distance of $0.01 \mathrm{~m}$ from the surface of the plate. Fig. 14 shows the sound power estimated using the identified radiation resistance matrix, $\widehat{\mathbf{R}}_{p}$, plotted along with the directly evaluated sound power. It can be seen from these results that the estimated sound power, again, is reasonably accurate. In this case, the majority of the resonance frequencies have been identified, however, the level of the sound power estimate, across the presented frequency range, is slightly less accurate than when calculated using the radiation resistance matrix that utilises the particle velocity 
in the identification process. This is due to the approximation used in the present method, which assumes that the structural velocity is equal to the acoustic particle velocity.

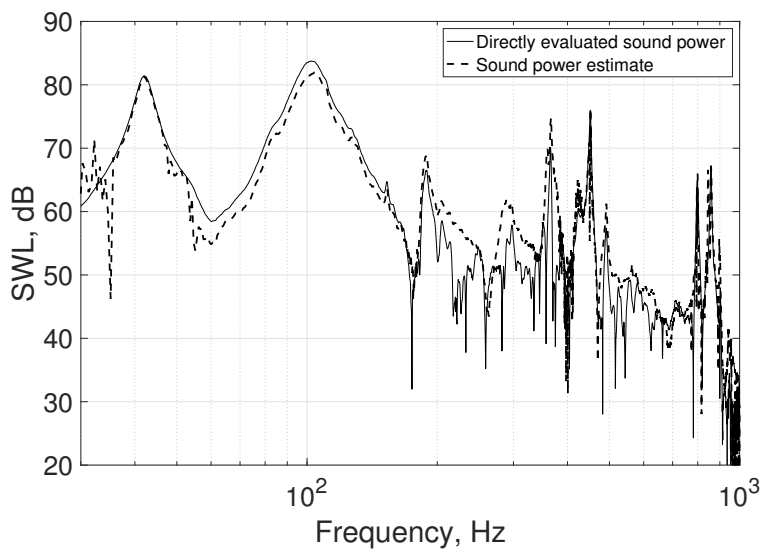

FIG. 14. Sound power radiated by the plate when acoustic excited with broadband noise. The solid line shows the measured sound power and the dashed line shows the sound power calculated using the proposed radiation resistance matrix $\widehat{\mathbf{R}}_{p}$.

\section{CONCLUSIONS}

The radiation resistance matrix allows for an accurate estimation of the sound radiation from a structure using only structural measurements. A number of formulations have been presented in previous work that enable the estimation and measurement of the radiation resistance matrix. However, these methods have not been widely applied to practical structures due to either limitations in their accuracy due to modelling requirements or the complexity of the measurement procedure for practical structures. Therefore, in this paper, a method of estimating the radiation resistance matrix from measurable structural and acoustic responses has been proposed. This method does not require a bespoke measurement 
device, does not require the measurements to be conducted in a free-field environment and is functional over the frequency range of interest for ASAC.

Two approaches to the radiation resistance matrix identification problem have been presented; the first requires measurement of both the acoustic particle velocity and structural velocity, whilst the second approach assumes that these are equal. Both methods are based on solving an inverse problem that is used to estimate the transfer response matrix between a number of structural and acoustic responses. In both methods, a series of response measurements are required when the structure is excited by a distribution of independent forces, but no knowledge about the operational structural excitation is assumed.

To assess the limits on the accuracy of the proposed radiation resistance matrix identification methods, a series of simulations for a flat rectangular plate have been carried out, as this provides a well-known benchmark. Within this framework, to investigate the practical requirements of the proposed estimation procedures, the numbers of forces and both acoustic and structural sensors used during the identification procedures were altered both independently and simultaneously. As the number of structural sensors used is decreased, the sound power level is overestimated at higher frequencies. As the number of forces used is decreased, the sound power level is underestimated at higher frequencies. In both instances, however, the resonance frequencies of the radiating modes are generally well estimated. As the number of acoustic sensors is decreased, the sound power estimate remains accurate over the presented frequency range, since the acoustic wavelengths within this range are much larger than the bending wavelengths in the plate and so sampling resolution limits are not met. 
Although the requirements in terms of the density of forces and sensors used in both methods are equivalent, it has also been shown that the second method, which makes the assumption that the structural velocity is equal to the particle velocity, is also sensitive to the distance of the acoustic sensor array from the structure. Specifically, as the acoustic pressure sensors are moved further away from the radiating surface, the accuracy of this identification method reduces and the sound power at frequencies where the distance between the surface and the acoustic pressure sensors is comparable to the acoustic wavelength is underestimated. Despite this limitation, this method may be more practicable in certain situations than the identification method that requires both acoustic pressure and particle velocity measurements.

Finally, to validate the simulation-based study, an experimental rig was set up to measure the radiation resistance matrix for a flat rectangular plate mounted on an enclosure. Based on the simulation study, three forces, structural sensors and acoustic sensors per the shortest acoustic wavelength were used to identify the radiation resistance matrix using the two approaches. This number was chosen as it provides a practicable configuration, but also provides insight into the practical accuracies and limitations of the proposed methods. The results of this experimental study have shown that the proposed identification methods able to accurately estimate both the level and resonance frequencies of the radiating modes up to around $400 \mathrm{~Hz}$, which corresponds to 8 forces/sensors per acoustic wavelength. At higher frequencies, the accuracy begins to reduce and, although the frequencies of the radiating modes are generally well estimated, errors in the estimated sound power level begin to occur. 


\section{ACKNOWLEDGMENTS}

This research was partially supported by an EPRSC iCASE studentship (Voucher number 15220040) and an EPSRC Prosperity Partnership ( EP/S03661X/1). All data supporting this study are openly available from the University of Southampton repository at https://doi.org/10.5258/SOTON/D0879

\section{REFERENCES}

\section{REFERENCES}

${ }^{1}$ A. Roure and A. Albarrazin, "The remote microphone technique for the active noise cotnrol," in Active 99, International Symposium on Active Control of Sound and Vibration (1999), pp. $1233-1244$.

${ }^{2}$ S. Elliott and A. David, "A virtual microphone arrangement for local active sound control," in MoVic, International conference on Motion and Vibration Control (1992), pp. 1027 1031.

${ }^{3}$ D. Moreau, B. Cazzolato, B. Zander, and C. Petersen, "A review of virtual sensing algorithms for active noise control," Algorithms 1 69-99 (2008).

${ }^{4}$ J. Garcis-Bonito, S. Elliott, and C. Boucher, "Generation of zones of quiet using a virtual microphone arrangement," The Journal of the Acoustical Society of America 101(2), 34983516 (1997). 
${ }^{5}$ S. Elliott and J. Cheer, "Modelling local active sound control with remote sensors in spatially random pressure fields," The Journal of the Acoustical Society of America 137(4), 1936-1946 (2015).

${ }^{6}$ F. Fahy and P. Gardonio, Sound and structural vibration (Academic Press Oxford, 2007).

${ }^{7}$ W. T. Baumann, F.-S. Ho, and H. H. Robertshaw, "Active structural acoustic control of broadband disturbances," The Journal of the Acoustical Society of America 92(4), 1998 - 2005 (1992).

${ }^{8}$ J. Cheer and S. Daley, "Active structural acoustic control using the remote sensor method," Journal of Physics: Conference Series 744(1), 012184 (2016).

${ }^{9}$ D. R. Hendricks, W. R. Johnson, S. D. Sommerfeldt, and J. D. Blotter, "Experimental active structural acoustic control of simply supported plates using a weighted sum of spatial gradients," The Journal of the Acoustical Society of America 136(5), 2598-2608 (2014).

${ }^{10}$ P. Aslani, S. D. Sommerfeldt, and J. D. Blotter, "Active control of simply supported cylindrical shells using the weighted sum of spatial gradients control metric," The Journal of the Acoustical Society of America 143(1), 271-280 (2018).

${ }^{11}$ S. Elliott and M. E. Johnson, "Radiation modes and the active control of sound power," Acoustical Society of America 94(4), 2194-2204 (1993).

${ }^{12}$ J. B. Fahnline and G. H. Koopmann, "A lumped parameter model for the acoustic power output from a vibrating structure," The Journal of the Acoustical Society of America 100(6), $3539-3547(1996)$. 
${ }^{13}$ J. B. Fahnline and G. H. Koopmann, "Numerical implementation of the lumped parameter model for the acoustic power output of a vibrating structure," The Journal of the Acoustical Society of America 102(1), 179 - 192 (1997).

${ }^{14}$ G. H. Koopmann and J. B. Fahnline, Designing Quiet Structures A Sound Power Minimization Approach (Academic Press London, 1997).

${ }^{15}$ A. Berkhoff, "Broadband radiation modes: Estimation and active control," The Journal of the Acoustical Society of America 111(3), 1295-1305 (2002).

${ }^{16}$ P. Gardonio, Y. S. Lee, S. Elliott, and S. Debost, "Active control of sound transmission through a panel with a matched pvdf sensor actuator pair," in Active 99 (1999), pp. 341 $-354$.

${ }^{17} \mathrm{~K}$. Henrioulle and P. Sas, "Experimental validation of a collocated pvdf volume velocity sensor/actuator pair," Journal of Sound and Vibration 265, 489-506 (2003).

${ }^{18}$ L.E. Kinsler, A.R. Frey, J.V. Sanders and A.B. Coppens, Fundamentals of Acoustics (John Wiley and Sons, 1999).

${ }^{19}$ O. Heuss, "Identification of power transfer matrices for active structural acoustic contorl," The Twentieth International Congress on Sound and Vibration (2013).

${ }^{20}$ R. Pintelon and J. Schoukens, System Identification: A Frequency Domain Approach (John Wiley and Sons, 2012). 